\title{
Tangencies in invariant disk packings for certain planar piecewise isometries are rare
}

\author{
Peter Ashwin and Xin-Chu Fu \\ School of Mathematical Sciences, \\ Laver Building, \\ University of Exeter, \\ Exeter EX4 4QE, UK.
}

January 8, 2001

\begin{abstract}
For piecewise isometries there is a natural coding given by the itinerary of a trajectory between the pieces or atoms of the partition on which it is defined. The set of points with the same coding is referred to as a cell and under certain general conditions the periodically coded cells define an invariant set that is a disjoint union of disks.

In this paper we investigate properties of this invariant disk packing. For a oneparameter family of PWI on a torus, we prove that tangencies between disks in this packing are very rare; more precisely they occur on a set of parameter values that is at most countably infinite. If such packings are dense we show that they are maximal in a sense of measure. We provide examples to show that the packing may not be dense if there is continuity over boundaries in the partition, and also that the absence of tangencies in the packing does not necessarily imply that the complement of the packing has positive Lebesgue measure.
\end{abstract}

Key words: Piecewise isometry, discontinuous dynamical system, disk packing.

\section{Introduction}

Piecewise isometries (PWIs) show a rich range of dynamical behaviours under iteration $[1$, $12,8,9,7]$ and are a natural extension of the so-called interval exchange transformations. 
We define them as follows: Let $M$ be a compact region of the plane such that $M=\cup \overline{M_{k}}$ with $\left\{M_{k}\right\}_{k=1}^{N}$ disjoint convex open polygons. We say $f: M \rightarrow M$ is an orientation preserving invertible piecewise isometry (or just PWI) if $\left.f\right|_{M_{k}}$ is a planar orientation preserving isometry, and $f: M \rightarrow M$ is invertible. We refer to $M_{k}$ as the atoms of the partition defining the PWI. In the standard way we identify $\mathbf{R}^{2}=\mathbf{C}$ and note that any orientation preserving isometry can be written as a map of the form $x \mapsto r x+w$ with $|r|=1$ and $w \in \mathbf{C}$.

There is a full measure subset of $x \in M$ such that we can define a unique itinerary; this is a map $\iota: M \rightarrow \Sigma(N)$ with $\Sigma(N)$ the set of bi-infinite words with $N$ letters, defined by

$$
[\iota(x)]_{n}=k \quad \text { if and only if } f^{n}(x) \in M_{k} .
$$

We say $\mathbf{s} \in \Sigma(N)$ is admissible if there is an $x \in M$ such that $\iota(x)=\mathbf{s}$. A sequence $\mathbf{s}$ is periodic if there is an $n>0$ such that $s_{k+n}=s_{k}$ for all $k$; the smallest such $n$ is the period. The set of points with the same itinerary we refer to as a cell, i.e. given an admissible s we define $C(\mathbf{s})=\iota^{-1}(s)$. We write $\mathcal{P} \subset \Sigma(N)$ to be the set of periodic admissible itineraries. Using convexity of the atoms and the preservation of convexity under isometry one can show the following:

Proposition 1 [9, 10, 13, 3] The cells $C(\mathbf{s})$ are convex planar regions; these have interior if and only if $\mathbf{s} \in \mathcal{P}$. For all admissible $\mathbf{s} \notin \mathcal{P}$ the cell is either a point or a line segment.

We say a PWI is an irrational rearrangement if $\left.f\right|_{M_{k}}(x)=e^{i \theta} x+w_{k}$ and $\theta / \pi \notin \mathbf{Q}$.

Proposition $2[10,4]$ Any n-periodic cell for an irrational rearrangement is a disk. The $n^{\text {th }}$ iterate of the map acts as an irrational rotation on the disk, i.e. its centre is an $n$-periodic point and it is surrounded by a nested set of invariant circles.

Hence, for PWIs that are irrational rearrangements, the set of periodic cells defines a union of disjoint disks

$$
C=\bigcup_{\mathbf{s} \in \mathcal{P}} C(\mathbf{s})
$$

that we refer to as the invariant disk packing of $M$ induced by the piecewise isometry. The complement (or exceptional set [11]) is defined by

$$
C^{c}=M \backslash C
$$


and consists of points with aperiodic (or no) codings. As a consequence, it consists of points whose trajectories accumulate on boundaries of the partition. If the invariant disk packing induced by a PWI is dense, we show in Corollary 1 that the packing is uniquely defined by the periodic orbits of the map.

It is a surprisingly difficult problem to identify the set of periodic admissible codings [13]; the thesis of Vowden studies a number of families of periodic codings for a specific map [14] and finds parameter regions of admissibility for several families of periodic sequences. However, the only case with a relatively complete understanding (and a description in terms of a substitition system) is the case with $\theta_{k}=\pi / 4$ [1]. In fact, for a typical piecewise isometry it is not even known whether $C$ has an infinite or finite number of connected components or even if it is non-empty. Figure 1 illustrates the numerical approximation of a disk packing for a typical parameter of the overflow oscillation map; see $[4,7]$ for more details.

In this paper we examine examples that enlighten the basic geometric properties of such invariant disk packings. Section 2 explores dense packings and those of maximal measure. We relate properties of the dynamics of PWIs to properties of the packing. More precisely, we conjecture that typically, the packing is dense but does not possess full measure and the packing graph (the graph defined by tangencies) is totally disconnected. We finish this section by giving a construction of a dense disk packing of a triangle with no tangents but zero measure complement.

In Section 3 we prove in Theorem 3 for a particular one-parameter family $f_{\theta}$ of PWIs on the torus (the so-called overflow oscillation map) that there are no tangencies in the disk packing for almost all values of $\theta$. We believe that this is a general property of families of invariant disk packings and gives some support to the conjectured behaviour discussed in Section 2. There is work in progress to generalize the method of proof to other families of PWIs. One major obstacle is that the general structure of parametrized families of PWIs is still poorly understood [5].

In Section 4 we show how to construct classes of PWIs with an invariant disk packing that is not dense; we also show how tangencies can be broken by introduction of extra atoms in the partition. These are discussed further in Section 5. 


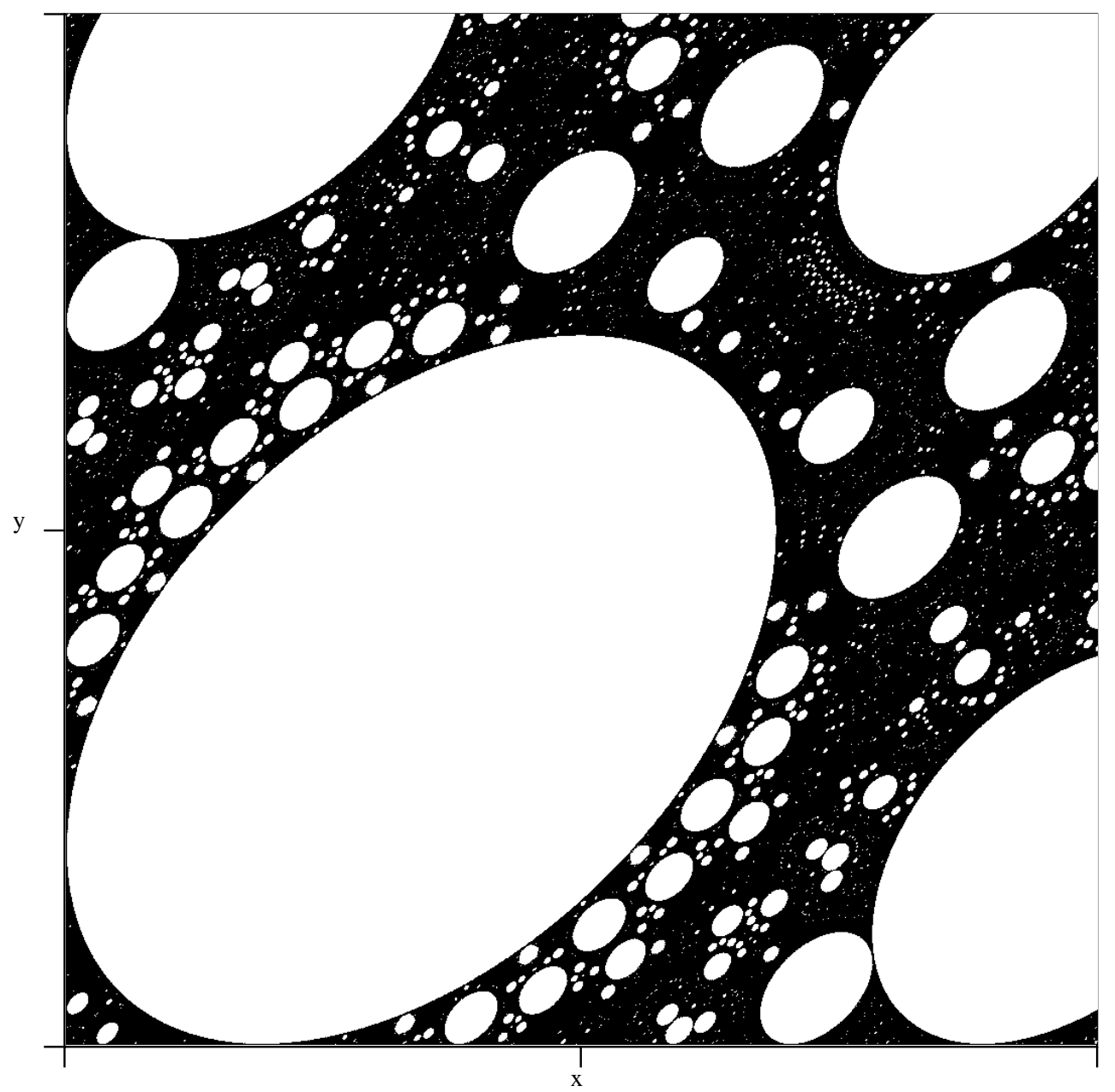

Figure 1: Numerical approximation of a detail of the invariant circle packing for a discontinuous map of the torus that is equivalent to a piecewise isometry under a linear shear of the domain; hence the invariant ellipses become invariant circles after the shear. The white regions correspond to periodically coded points while the black complement consists of aperiodically coded points. The region shown is the square $[-1,-0.875]^{2}$ of the phase space $[-1,1]^{2}$ for the map $(x, y) \mapsto(y, g(-x+2 y \cos \theta))$ where $g(y)$ is the discontinuous function that is the identity on $[-1,1)$ and is periodic with period 2 . In this case $\theta=\cos ^{-1}(0.45)$; similar pictures are found for other $\theta$ as long as $\theta / \pi$ is irrational. 


\section{Disk packings}

We define $B_{r}(x)=\{y \in \mathbf{C}:|x-y|<r\}$ and say a set $X \subset M \subset \mathbf{C}$ is a disk packing of $M$ if

$$
X=\bigcup_{i=1}^{N} B_{r_{i}}\left(x_{i}\right)
$$

where $B_{r_{i}}\left(x_{i}\right)$ are disjoint (non-overlapping) open disks with radii $r_{i}>0$ and centres $x_{i} \in \mathbf{C}$ ( $N$ may be infinite). There is a one-to-one correspondence between disk packings and circle packings by circles of radius $r_{i}$, centre $x_{i}$. A disk packing is said to be dense if $\bar{X}=\bar{M}$.

Such a packing is defined, up to permutation of the indices, by the pairs

$$
\left\{\left(x_{i}, r_{i}\right): i=1, \cdots, N \leq \infty\right\}
$$

with the constraints (non-empty) $r_{i}>0$, (containment) $B_{r_{i}}\left(x_{i}\right) \subset M$ for all $i$ and (no overlap) $\left|x_{i}-x_{j}\right| \geq r_{i}+r_{j}$ for all $i \neq j$. We refer to a disk packing satisfying these as $D P\left(\left\{\left(x_{i}, r_{i}\right)\right\}\right)$. The Lebesgue measure of this packing can be computed as

$$
\ell\left(D P\left(\left\{x_{i}, r_{i}\right\}\right)\right)=\pi \sum_{i} r_{i}^{2}
$$

We say a disk packing $\left\{\left(x_{i}, r_{i}\right)\right\}$ is maximal given centres $x_{i}$ if

$$
\ell\left(D P\left(\left\{\left(x_{i}, r_{i}\right)\right\}\right) \geq \ell\left(D P\left(\left\{\left(x_{i}, s_{i}\right)\right\}\right)\right)\right.
$$

for any other packing $\left\{\left(x_{i}, s_{i}\right)\right\}$ with the same centres. We say a disk packing $D P$ of $M$ is dense if $\overline{D P}=M$.

Theorem 1 Suppose that $D P\left(\left\{\left(x_{i}, r_{i}\right)\right\}\right)$ is dense in $M$, then it is maximal and is determined uniquely by the $x_{i}$.

Proof Suppose that a disk packing $\left\{\left(x_{i}, r_{i}\right)\right\}$ is dense, then the radii are given by $r_{i}=$ $\inf _{j \neq i}\left|x_{j}-x_{i}\right|$. To see this, let $R_{i}=\inf _{j \neq i}\left|x_{j}-x_{i}\right|$. If $r_{i}>R_{i}$ then there will be an overlap between disk $i$ and some other disk. If $r_{i}<R_{i}$ then for any $\rho$ with $r_{i}<\rho<R_{i}$ the disk $B_{\rho}\left(x_{i}\right)$ will intersect at most finitely many other disks in the packing. Hence the packing cannot be dense in the set $B_{\rho}\left(x_{i}\right) \backslash B_{r_{i}}\left(x_{i}\right)$ and by contradiction $r_{i}=R_{i}$. Maximality comes from noting that if $0<s_{i} \leq r_{i}$ then $\ell\left(D P\left(\left\{\left(x_{i}, s_{i}\right)\right\}\right)\right) \leq \ell\left(D P\left(\left\{\left(x_{i}, r_{i}\right)\right\}\right)\right)$.

The next result relates the dynamics of a PWI to its invariant disk packing. 
Corollary 1 If the invariant disk packing $C$ defined by a $P W I f$ is dense, then the set of periodic orbits uniquely defines the centres of disks for this disk packing.

The following lemma will be of use in later sections.

Lemma 1 Suppose that two disks in the invariant disk packing $C$ are tangent. Then one can find two disks $C_{1}, C_{2}$ in $C$ that are tangent and such that and $C_{1} \subset M_{1}, C_{2} \subset M_{2}$ where $M_{1}, M_{2}$ are atoms in the partition defining the piecewise isometry.

Proof Given any two tangent disks they must have distinct periodic codings with periods $n_{1}$ and $n_{2}$ say. By taking images of the pair, after a finite number of iterates (less than $\left.\min \left(n_{1}, n_{2}\right)\right)$ they must land in different atoms of the partition.

The packing graph A disk packing $\left\{\left(x_{i}, r_{i}\right)\right\}$ has a tangency between disks $i \neq j$ if and only if $\left|x_{i}-x_{j}\right|=r_{i}+r_{j}$. This naturally defines a packing graph where vertices correspond to disks and edges correspond to tangencies between disks. A disk packing is said to be tangent free if this graph has no edges.

There is a particularly well-studied disk packing; the Apollonian disk packing of the unit disk (see [2] for an excellent review, recent results and a discussion of open questions). This packing is such that every disk inscribes a curvilinear triangle comprised of the space between three other disks that are mutually tangent.

An Apollonian packing of a region $M$ can similarly be defined to be a dense packing such that every disk as either tangent to $\partial M$ and two others that are also tangent to $\partial M$ or as above. This is clearly dense by construction; Apollonian packings have packing graphs that have the maximum degree possible of connectivity as every vertex has an infinite number of neighbours.

There are numerical estimations of the Hausdorff dimension of the complement of Apollonian packings that indicate that they have dimension 1.3056867. A conjecture of Boyd [6] surmises that Apollonian packings are maximal in another sense; namely that they have complements with minimal Hausdorff dimension, i.e. any other packing must have a complement with Hausdorff dimension that is at least this value of 1.3056867 . Lower bounds have been found [2] but these remain much lower than this amount. 


\subsection{Example of a tangent-free dense disk packing with zero measure complement}

One might surmise that a tangent free disk packing necessarily has less than full measure. In this section we construct a tangent-free dense disk packing with zero measure complement to show that this is not the case. The construction is shown diagrammatically in Figure 2. We consider an 'upwards' equilateral triangle $T_{1}$ and pack this with its maximal inscribed disk $C_{1}$ and in infinite number of 'downwards' equilateral triangles $\left\{T_{1, i}\right\}_{i=1}^{\infty}$ as shown.

We then repeat the process by filling each 'downwards' triangle $T_{1, i}$ with its maximal inscribed disk $C_{1, i}$ and a similar copy of the triangle $T_{1}$, now 'upwards' $\left\{T_{1, i, j}\right\}_{j=1}^{\infty}$ by similarities $S_{i}$ such that $S_{i}\left(T_{1}\right)=T_{1, i}$. These triangles are uniquely defined by placing the triangle within each curvilinear triangle such that the line from where the curved side meets the vertex of the new triangle to the opposite vertex bisects the angle there.

This process is repeated on each triangle to get a packing with a countable number of disks $C_{1, i_{1}, i_{2}, \cdots, i_{n}}$, and we write $C$ to denote the union of all such disks in the packing. At each step all points are either within a curvilinear triangle with at most one curved side that will be subdivided, or in a disk. Hence one can see that the set of disks constructed is dense in the original $T_{1}$. Moreover, note that any two disks in the packing are inscribed in different triangles, and because of alternating generations of 'upwards' and 'downwards' triangles, triangles $T_{1, i_{1}, i_{2}, \cdots, i_{n}}$ can only touch with the vertex of one against the side of the other. Hence there can be no tangencies between disks in $C$.

Theorem 2 This packing is full measure, i.e. $\ell\left(T_{1}\right)=\ell(C)$.

Proof We define $D_{1}=C_{1}, D_{n+1}=D_{n} \cup\left(\cup_{i} S_{i}\left(D_{n}\right)\right)$ and note that $C=\cup_{n} D_{n}$. Now define $P=\ell(C) / \ell\left(T_{1}\right)$ to be the proportion of $T_{1}$ taken up by the disk packing. By construction we see that

$$
\ell\left(T_{1}\right)=\ell\left(C_{1}\right)+\sum_{i} \ell\left(T_{1, i}\right)
$$

as the complement of $\cup T_{1, i}$ in $T_{1} \backslash C_{1}$ are a countable union of Sierpinski gaskets and as such have zero measure. The uniform scaling of meausure of the similarities $S_{i}$ implies that $P=\ell\left(C \cap T_{1, i}\right) / \ell\left(T_{1, i}\right)$ for all $i$, and so

$$
\ell(C)=\ell\left(C_{1}\right)+\sum_{i} \ell\left(C \cap T_{1, i}\right)=\ell\left(C_{1}\right)+P \sum_{i} \ell\left(T_{1, i}\right)
$$




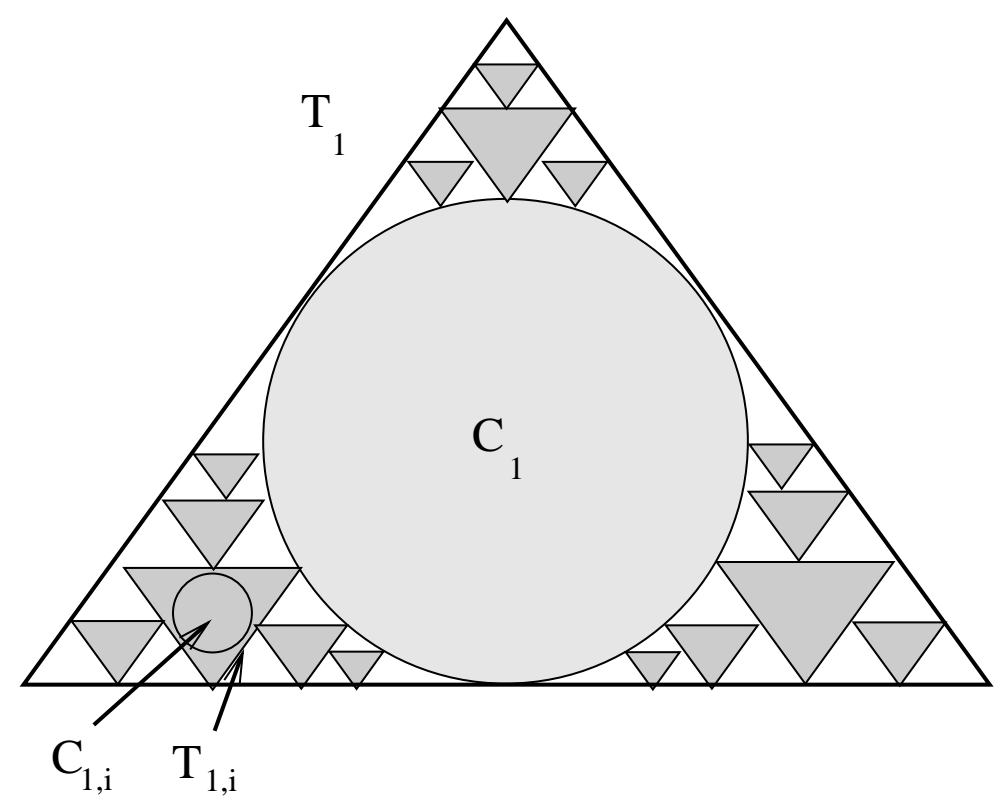

Figure 2: The construction of a tangent-free dense disk packing of the triangle $T_{1}$ with zero measure complement. The whole of $T_{1}$ is repeatedly mapped into each $T_{1, j}$ using similarities to give a union of disks that are images of $C_{1}$ and which pack $T_{1}$ densely, using full measure and with no tangencies between disks.

which implies that $P \ell\left(T_{1}\right)=\ell\left(C_{1}\right)+\sum_{i} P \ell\left(T_{1, i}\right)$. This, together with the other expression for $\ell\left(T_{1}\right)$ implies that either $\ell\left(C_{1}\right)=0$ (which is plainly a contradiction) or $P=1$ which gives the required result.

The complement of the packing $C$ can be generated as the limit set of the countable set of the similarities $\left\{S_{i}\right\}_{i=1}^{\infty}$. Note that copies of the Sierpinsky gasket can be found as subsets of $C$, implying that $\operatorname{dim}_{H}(C) \geq \frac{\log 3}{\log 2}$.

\section{Genericity of tangent-free packings for a piecewise isom- etry of the torus.}

In this section we prove that tangencies are extremely rare for a particular one parameter family of PWIs of the torus known as the lossless overflow oscillation problem. This map is usually written as a linear map of the square $[-1,1)^{2}$ to itself, and an numerically obtained circle packing is shown in Figure 1. The map is equivalent to the following 


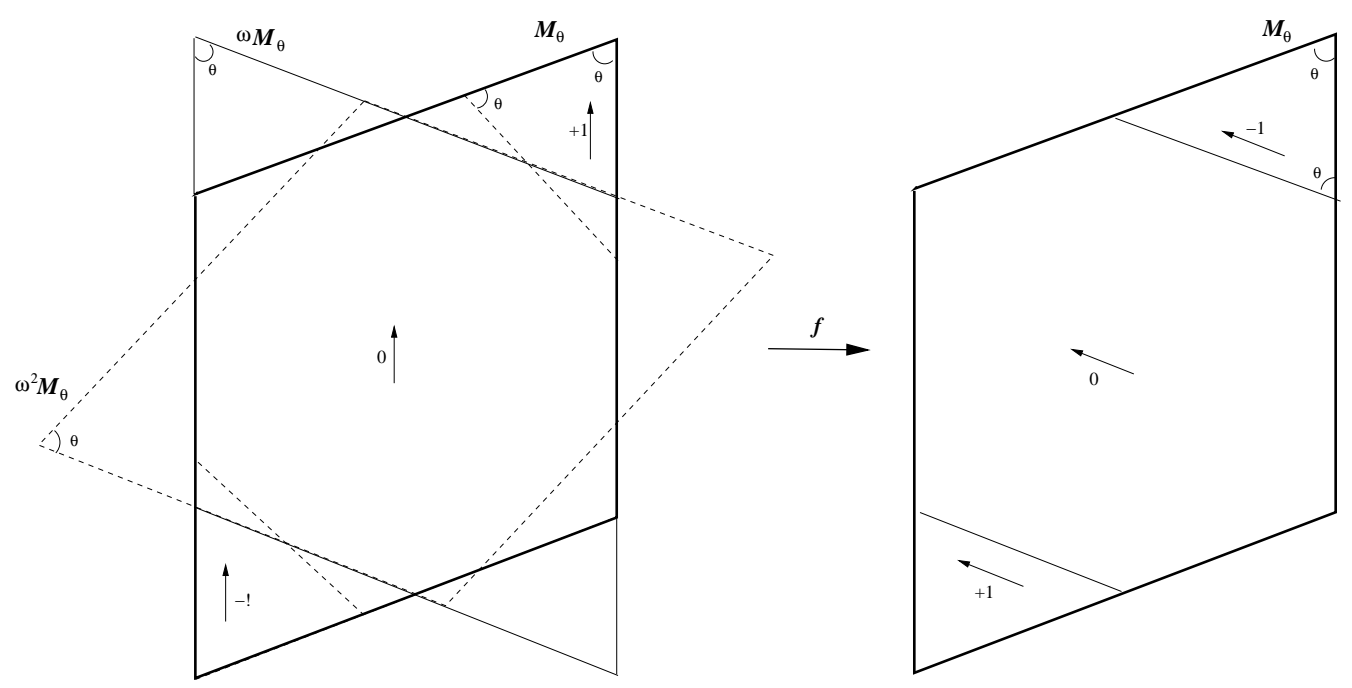

Figure 3: The rhombus on which the piecewise isometry of the torus is defined. The image of the partition into two triangles and a hexagon is shown on the right. There is a one-parameter of such maps parameterized by the angle $\theta$ of the rhombus. We write $\omega=e^{i \theta}$.

piecewise isometry after a linear change of coordinates.

Fix $\theta \in(0, \pi)$ and consider the rhombus unit cell for a torus:

$$
M_{\theta}=\left\{z \in \mathbf{C}:|\operatorname{Re}(z)| \leq 1 \text { and }\left|\operatorname{Re}\left(z e^{i \theta}\right)\right| \leq 1\right\}
$$

illustrated in Figure 3. We define $f: M_{\theta} \rightarrow M_{\theta}$ by

$$
f(z)=e^{i \theta} z+W k(z)
$$

where $W=-\frac{2 i}{\sin \theta}$ is a constant and

$$
k(z)=\left\{\begin{aligned}
+1 & \text { if } \operatorname{Re}\left(z e^{2 i \theta}\right)>1 \\
-1 & \text { if } \operatorname{Re}\left(z e^{2 i \theta}\right) \leq-1 \\
0 & \text { otherwise }
\end{aligned}\right.
$$

Obviously, $k(z)$ identifies the partition elements $\{-1,0,+1\}$ and we use this coding. It is clear from the geometry that, disregarding the discontinuities, the mapping is invertible. The main result of this section is the following:

Theorem 3 There is a countable set of $\theta \in[0,2 \pi)$ for which there are tangencies between any periodic disks in the disk packing $C$ induced by $f_{\theta}$. Therefore there is a full measure set of $\theta$ such that the disk packing $C$ has no tangencies. 
We will prove this by showing that tangencies can only occur at isolated parameter values. Let $\omega=e^{i \theta}$ and consider any periodic coding (we do not assume at this stage that it is admissible) $\mathbf{k}$ of minimal period $n$ where $k_{j} \in\{-1,0,+1\}$ such that

$$
\mathbf{k}=P\left(k_{0} k_{1} k_{2} \cdots k_{n-1}\right)
$$

where $P$ denotes the periodic concatenation of its argument. If this is admissible then there will be a unique periodic point $z=z_{\mathbf{k}}$ with $\iota(z)=\mathbf{k}$ satisfying

$$
z=\omega^{n} z+W \sum_{j=0}^{n-1} \omega^{j} k_{n-j-1}
$$

This means that

$$
z_{\mathbf{k}}=\frac{W}{1-\omega^{n}} \sum_{j=0}^{n-1} \omega^{j} k_{n-j-1} .
$$

If we write $\eta=e^{i t}$ and $\theta=2 t$ then this can be written as

$$
z_{\mathbf{k}}=\frac{W}{\left(\eta^{-n}-\eta^{n}\right)} \sum_{j=0}^{n-1} \eta^{2 j-n} k_{n-j-1}=\frac{1}{\sin 2 t \sin n t} \sum_{j=0}^{n-1} \eta^{2 j-n} k_{n-j-1} .
$$

Lemma 2 Suppose that $\mathbf{k}, \ell \in \mathcal{P}$ are nonidentical admissible periodic sequences. Then the largest inscribed disks of these cells with these codings are tangent for at most a finite number of values of $\theta$. In consequence, there are at most finitely many irrational $\theta$ at which the disks $C(\mathbf{k})$ and $C(\ell)$ are tangent.

Proof Suppose that the two disks $C(\mathbf{k})$ and $C(\mathbf{m})$ are tangent. If $k_{0}=m_{0}$ then they are in the same atom and so their images under $f$ will still be tangent, hence we can assume without loss of generality (by iterating if necessary) that $k_{0} \neq m_{0}$.

The tangencies must occur at the tangencies of the atoms, and hence on the lines where $\operatorname{Re}\left(\omega^{2} z\right)= \pm 1$. Since the centres of the disks are given by $z_{\mathbf{k}}$ and $z_{\mathbf{m}}$ we see that whenever there is a tangency

$$
\Phi=\operatorname{Im}\left(\omega^{2}\left(z_{\mathbf{k}}-z_{\mathbf{m}}\right)\right)=0
$$

although note that this condition is not sufficient for a tangency. Writing

$$
\phi=\Phi \sin \theta
$$

to remove the $\theta$ dependent term of $W$, and using (1) we see that the left hand side can be computed as

$$
\phi=\operatorname{Im}\left(\frac{1}{\sin n t} \sum_{j=0}^{n-1} \eta^{4+2 j-n} k_{n-j-1}-\frac{1}{\sin m t} \sum_{j=0}^{m-1} \eta^{4+2 j-m} m_{m-j-1}\right)
$$


and so

$$
\phi=\frac{1}{\sin n t} \sum_{j=0}^{n-1} k_{n-j-1} \sin ((4+2 j-n) t)-\frac{1}{\sin m t} \sum_{j=0}^{m-1} m_{m-j-1} \sin ((4+2 j-m) t)
$$

where $t=\theta / 2$. Note that we can write $\phi(\eta)$ as a ratio of polynomials of order at most $2 n m$. Therefore either $\phi$ is identically zero for all $\eta \in \mathbf{C}$, or it has a finite number of zeros corresponding to zeros of the numerator. In particular, if it is not identically zero then it can only have a finite number of zeros on $|\eta|=1$. Writing $\phi=p(t) / q(t)$ as a rational trigonometric polynomial we see that

$$
p(t)=\sum_{j=0}^{n-1} k_{n-j-1} \sin m t \sin (4+2 j-n) t-\sum_{j=0}^{m-1} m_{m-j-1} \sin n t \sin (4+2 j-m) t .
$$

If $m=n$ we can remove the factor $\sin m t$ and then note that this is identically zero if and only if

$$
\sum_{j=0}^{n-1}\left(k_{n-j-1}-m_{n-j-1}\right) \sin (4+2 j-n) t
$$

is identically zero. Observe for all $n \geq 1$, the highest frequency term here is $\left(k_{0}-m_{0}\right) \sin (2+$ $n) t$. Since this cannot be cancelled by sums of lower frequency terms, this can only happen if $k_{0}=m_{0}$, giving a contradiction.

If $m \neq n$ we can expand $p(t)$ in terms of a sum of $\cos k t$ terms. Identifying the terms multiplying $k_{0}$ and $m_{0}$ we have $k_{0} \sin m t \sin (2+n) t-m_{0} \sin n t \sin (2+m) t$ which, when expanded, gives rise to a highest frequency term of the form

$$
\left(m_{0}-k_{0}\right) \cos (2+n+m) t .
$$

As above this cannot be cancelled by other cos $k t$ terms as these have $|k|<2+n+m$. Therefore, $p$ identically zero implies that $k_{0}=m_{0}$, which is again a contradiction.

We are now in a position to prove the main result of this section.

Proof [of Theorem 3] If we consider the set of all possible periodic sequences of the three symbols $\{-1,0,+1\}$, this is countable, as is the set of pairs of non-identical periodic sequences. For any such pair $(\mathbf{k}, \mathbf{m})$ Lemma 2 shows that there are at most a finite number of $\theta$ at which tangencies can occur. Hence the set of $\theta$ for which there are tangencies is at most countably infinite. 
The proof relies on the fact that the centres of the disks can be found explicitly as nontrivial analytic functions of the parameter. It is probable that such a proof can be adapted to show that there are no tangencies for certain other affine maps, e.g. of the form examined in [10]. However, at present there is not enough known about more general families of piecewise isometries (such that the number of atoms and their faces are preserved) [5] to be able to apply a similar method.

\section{Changing the partition}

In this section we give two constructions. The first shows how to construct a disk packing that is not dense while the second shows how tangencies can easily be broken by introduction of new atoms in the partition.

Example of a PWI such that $C$ is not dense. We demonstrate that one can easily perturb the partitions of PWIs such that the set of admissible periodically coded points is not dense, simply by subdividing an atom containing a periodic island that is rotating irrationally.

Consider any invertible piecewise isometry $f: M \rightarrow M$ with irrational rotations on some partition $\left\{M_{k}\right\}_{k=1}^{n}$ of $M$. We pick any atom, say $M_{n}$ that is hit by at least one periodic disk $C(\mathbf{k})$ with coding $\mathbf{k}$. We split this atom into two to give a new partition $\left\{N_{k}\right\}_{k=1}^{n+1}$ where

$$
N_{k}=M_{k} \text { for } 1 \leq k<n, \quad N_{n} \cup N_{n+1} \subset M_{n}
$$

such that the line segment $L=\bar{N}_{n} \cap \bar{N}_{n+1}$ intersects $C(\mathbf{k})$. Now consider the periodically coded set $C$ defined by the same map $f$ but using this new partition. All periodic codings that were admissible and do not include $M_{n}$ remain as before. If we consider the disk $C(\mathbf{k})$, some iterate of the map acts as an irrational rotation on the disk and so iterates of the line $L \cap C(\mathbf{k})$ contained in the discontinuity set will be dense in an open annulus $A \subset C(\mathbf{k})$ (see Figure 4). All points in $A$ will have coding that is aperiodic. Therefore $D \supset A$ and $C$ is not dense.

The following result is much weaker than that obtained for the overflow oscillation map, but at least it shows that tangencies are easily destroyed in more general PWIs.

Lemma 3 Suppose that we have an irrational rearrangement such that two periodically 


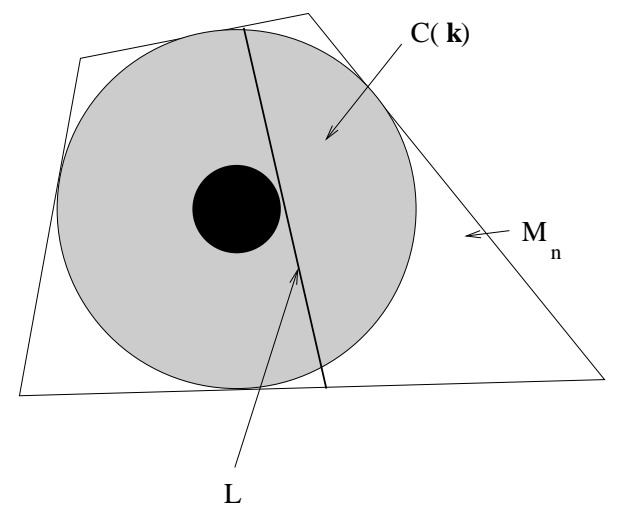

Figure 4: Construction of a PWI such that $C$ is not dense. Suppose that a PWI has a disk $C(\mathbf{k})$ of periodically coded points on which the first return is an irrational rotation in the atom $M_{n}$. By splitting this atom along the line $L$ we ensure that all points in the annulus shaded grey are aperiodically coded and therefore $C$ avoids this annulus.

coded disks $C_{1}$ and $C_{2}$ are tangent. Then by adding new partitions, one can find an open set of nearby PWIs (in the topology induced by measuring the $L_{1}$ norm of the difference between the maps) such that $C_{1}$ and $C_{2}$ are no longer tangent.

Proof Without loss of generality we can assume that $C_{1}$ and $C_{2}$ have centres on the real axis with common tangent that is the imaginary axis. The centres of $C_{i}$ we take to be $z_{i}$ with $z_{1}<0$ and $z_{2}>0$. Note that $e^{i \phi_{i}} z_{i}+w_{i}=z_{i}$ for some $\phi_{i}=n_{i} \theta$ and $w_{i}$ determined only by the coding and the piecewise isometry. According to Lemma 1, we can assume $C_{1} \subset M_{1}, C_{2} \subset M_{2}$ where $M_{1}, M_{2}$ are atoms in the partition defining the piecewise isometry.

If we can perturb the isometry to destroy tangency while preserving the invertibility, then we are done. If not we only need to perturb one of the atoms $M_{1}$ and $M_{2}, M_{1}$ say, to destroy the tangency between $C_{1}$ and $C_{2}$. The perturbation can be done in the way shown in Figure 5.

We define a new piecewise isometry $g: M \rightarrow M$ as follows: $g(x)=f(x)$ for $x \notin C_{\varepsilon}$, where $C_{\varepsilon}$ is the thin "loop" in Figure 5 (a); when $x$ is in a corner of $C_{\varepsilon}$ (see Figure $5(\mathrm{~b})$ ), we define $g(A)=f(B), g(B)=f(A), g(C)=f(D), g(D)=f(C), g(E)=f(F)$, $g(F)=f(E)$; when $x$ is in the thin rectangles of $C_{\varepsilon}$ in Figure 5 (a), we define $g(x)=f\left(x^{\prime}\right)$, where $x^{\prime}$ is a point shifted a certain distance clockwise along the "loop" consisting only the thin rectangles of $C_{\varepsilon}$ in Figure 5 (a). $g$ is a invertible piecewise isometry over a partitiion 


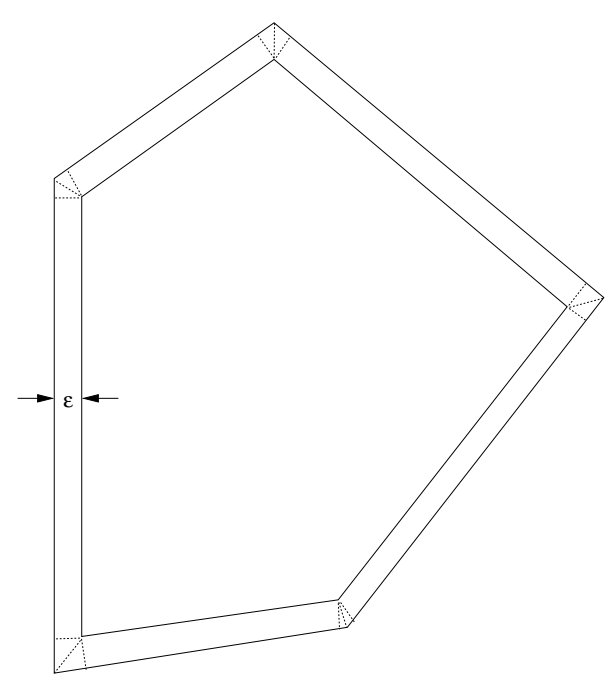

(a)

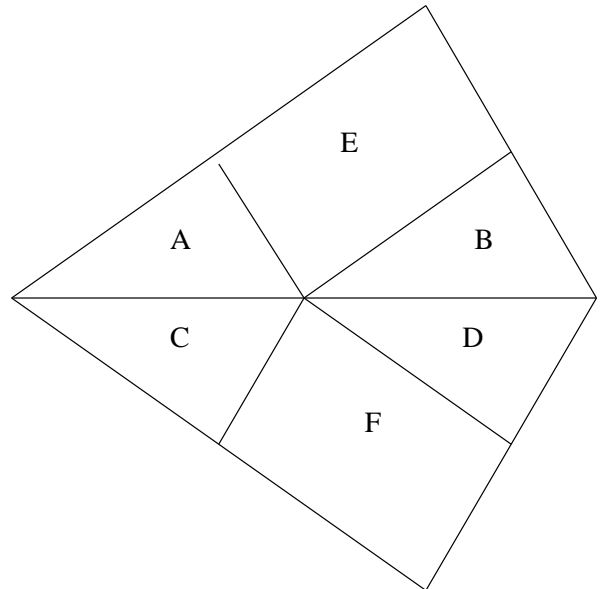

(b)

Figure 5: The perturbation of a piecewise isometry. (a) Shows how to perturb the atom $M_{1}$. (b) Shows how to deal with the corners in (a), where $A, B, C, D$ are congruent triangles, and $E, F$ are congruent rectangles.

with more atoms. When $\varepsilon$ is small enough, $g$ will be arbitrarily close to $f$ except on a set of arbitrarily small measure.

\section{Discussion}

The invariant disk packings induced by planar piecewise isometries are remarkable and compelling subsets of the plane. We have shown here that at least for one particular family of such maps, these packings are loose in the sense that they are tangent free. This is consistent with the conjecture that they do not have full Lebesgue measure.

It would be interesting to be able to characterize any self-similar structure of the dynamically defined packing; this has been done for a very particular map in [1]. For smooth maps, renormalization provides a useful tool in the study of self-similar behaviour of an area-preserving maps and universal behaviour of a class area-preserving maps. If a map $f$ converges under renormalization to a fixed point of the renormalization operator, $f$ would have asymptotically self-similar behaviour on long timescales and small space scales. For the tangent-free packing constructed in Section 2.1 there are exact self similarities. 
However, the evidence is that any self similarity for the dynamically defined disk packings is likely to be much more subtle.

\section{Acknowledgement}

We thank the EPSRC for support via grant GR/M36335, and Arek Goetz and Matthew Nicol for discussions concerning this research.

\section{References}

[1] R. Adler, B. Kitchens and C. Tresser, Dynamics of nonergodic piecewise affine maps of the torus. Preprint, 1999.

[2] D. Aharonov \& K. Stephenson, Geometric sequences of disks in the Appolonian packing. Algebra i Analiz 9:104-140 (1997).

[3] P. Ashwin, Elliptic behaviour in the sawtooth standard map, Phys. Lett. A 232, 409 (1997).

[4] P. Ashwin, X.-C. Fu and J.R. Terry, Riddling of invariant sets for some discontinuous maps preserving Lebesgue measure. preprint (2000).

[5] P. Ashwin and X.-C. Fu, On the geometry of planar piecewise isometries. in preparation (2001).

[6] D.W. Boyd, The residual set dimension of the Apollonian disk packing. Mathematika 20:170-174 (1973).

[7] A.C. Davies, Nonlinear oscillations and chaos from digital filter overflow. Phil. Trans. R. Soc. Lond. A 353:85-99 (1995).

[8] A. Goetz, Perturbation of 8-attractors and births of satellite systems, Intl. J. Bifn. Chaos 8, 1937 (1998).

[9] A. Goetz, Dynamics of a piecewise rotation. Continuous and Discrete Dynamical Systems 4:593-608 (1998).

[10] A. Goetz, Stability of cells in non-hyperbolic piecewise affine maps and in piecewise rotations. To appear in Nonlinearity (2000). 
[11] A. Goetz, Dynamics of a piecewise isometries. Illinois J. Math. 44:465-478 (2000).

[12] E. Gutkin and H. Haydn, Topological entropy of generalized polygon exchanges. Bull. Amer. Math. Soc. 32:50-57 (1995).

[13] L. Kocarev, C.W. Wu and L.O. Chua, Complex behaviour in Digital filters with overflow nonlinearity: analytical results. IEEE Trans CAS-II 43:234-246 (1996).

[14] C.J. Vowden, PhD thesis, Department of Engineering, University of Warwick, UK (1999). 